\title{
openheart SuPAR predicts postoperative complications and mortality in patients with asymptomatic aortic stenosis
}

\author{
Gethin W Hodges, ${ }^{1}$ Casper N Bang, ${ }^{2}$ Jesper Eugen-Olsen, ${ }^{3}$ Michael H Olsen, ${ }^{4,5}$ \\ Kurt Boman, ${ }^{6}$ Simon Ray, ${ }^{7}$ Antero Y Kesäniemi, ${ }^{8}$ Jørgen L Jeppesen, ${ }^{1}$ \\ Kristian Wachtell ${ }^{9}$
}

To cite: Hodges GW, Bang CN, Eugen-Olsen J, et al. SuPAR predicts postoperative complications and mortality in patients with asymptomatic aortic stenosis. Open Heart 2018;5:e000743. doi:10.1136/ openhrt-2017-000743

Received 31 October 2017 Revised 30 November 2017 Accepted 20 December 2017

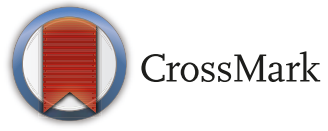

For numbered affiliations see end of article.

\section{Correspondence to} Dr Gethin W Hodges; gethin. william.hodges.02@regionh.dk

\section{ABSTRACT}

Background We evaluated whether early measurement of soluble urokinase plasminogen activator receptor (suPAR) could predict future risk of postoperative complications in initially asymptomatic patients with mild-moderate aortic stenosis (AS) undergoing aortic valve replacement (AVR) surgery.

Methods Baseline plasma suPAR levels were available in 411 patients who underwent AVR surgery during followup in the Simvastatin and Ezetimibe in Aortic Stenosis (SEAS) study. Cox analyses were used to evaluate suPAR in relation to all-cause mortality and the composite endpoint of postoperative complications (all-cause mortality, congestive heart failure, stroke and renal impairment) occurring in the 30-day postoperative period.

Results Patients with initially higher levels of suPAR were at increased risk of postoperative mortality with a HR of $3.5(95 \% \mathrm{Cl} 1.4$ to $9.0, \mathrm{P}=0.008)$ and postoperative complications with a HR of $2.7(95 \% \mathrm{Cl} 1.5$ to 5.1 , $\mathrm{P}=0.002)$, per doubling in suPAR. After adjusting for the European System for Cardiac Operative Risk Evaluation or Society of Thoracic Surgeons risk score, suPAR remained associated with postoperative mortality with a HR 3.2 (95\% Cl 1.2 to 8.6, $\mathrm{P}=0.025)$ and $2.7(95 \% \mathrm{Cl} 1.0$ to 7.8 , $\mathrm{P}=0.061)$; and postoperative complications with a HR of $2.5(95 \% \mathrm{Cl} 1.3$ to $5.0, \mathrm{P}=0.007)$ and $2.4(95 \% \mathrm{Cl} 1.2$ to $4.8, \mathrm{P}=0.011$ ), respectively.

Conclusion Higher baseline suPAR levels are associated with an increased risk for postoperative complications and mortality in patients with mild-moderate, asymptomatic AS undergoing later AVR surgery. Further validation in other subsets of AS individuals are warranted.

Trial registration number NCT00092677; Post-results.

\section{INTRODUCTION}

Soluble urokinase plasminogen activator receptor (suPAR) is a biomarker of inflammation that is associated with adverse prognosis in a range of diseases, including cancer, cardiovascular, renal and liver disease. ${ }^{1-4}$ SuPAR appears to reflect a chronic atherosclerotic disease burden and subclinical organ damage $^{1}$ and was recently shown to predict cardiovascular events and mortality in individuals with asymptomatic aortic stenosis (AS). ${ }^{5}$ Furthermore, suPAR has been shown

\section{Key questions}

What is already known about this subject?

- The inflammatory marker soluble urokinase plasminogen activator receptor (suPAR) is of prognostic value in a range of cardiovascular diseases, including in initially asymptomatic patients with mild-moderate aortic stenosis.

What does this study add?

- Patients with initially higher levels of suPAR undergoing aortic valve replacement surgery are at increased risk of postoperative complications and mortality.

How might this impact on clinical practice?

- SuPAR appears to provide additive prognostic information beyond the European System for Cardiac Operative Risk Evaluation II and Society of Thoracic Surgeons score; however, further validation in other patient groups is needed.

to be predictive of acute kidney injury in patients undergoing cardiac surgery, even in patients without pre-existing chronic kidney disease. $^{6}$

In apparently asymptomatic patients with AS, the potential benefits of an aortic valve replacement (AVR) procedure must be weighed against the risks of surgery. ${ }^{78}$ Validated risk stratification tools such as the European System for Cardiac Operative Risk Evaluation (EuroSCORE) II and Society of Thoracic Surgeons (STS) score are useful in predicting early 30-day mortality, and more recently, the prognostic utility of alternative scoring systems incorporating inflammatory markers has been demonstrated. ${ }^{9-14}$

We, therefore, hypothesised that suPAR would be of prognostic value in predicting early (30 day) postoperative AVR mortality and complications in patients with mild-moderate, asymptomatic AS and furthermore would enhance the performance of the EuroSCORE II and STS risk-score models. 


\section{METHODS}

\section{Study design and patient population}

This study stems from the larger Simvastatin and Ezetimibe in Aortic Stenosis (SEAS) study, which evaluated the potential of lipid-lowering drug treatment to minimise aortic valve events, cardiovascular disease and death. The baseline characteristics and design protocol for the main SEAS study have been published in detail elsewhere. ${ }^{15}$ In brief, 1873 patients with mild-moderate AS, as determined by Doppler ultrasound (aortic peak velocity $\geq 2.5$ and $\leq 4.0 \mathrm{~m} / \mathrm{s}$ ), received treatment with either simvastatin $40 \mathrm{mg}$ and ezetimibe $10 \mathrm{mg}$ or placebo over a 4.3-year period. The original results showed that lipid-lowering treatment was not associated with a reduction in the primary and combined endpoint; however, a $22 \%$ reduction in ischaemic events was observed. ${ }^{15}$ In this substudy, a total of 545 patients underwent conventional AVR surgery during this follow-up period (repeat operations were excluded). Plasma suPAR levels at baseline were available in 411 of these patients. The median time from baseline suPAR measurement to AVR surgery was 994 days (IQR 637-1270 days). A postoperative follow-up period of 30 days was chosen to reflect the standard used by the EuroSCORE II and STS score. In total, 134 patients were excluded due to missing values (assumed to be missing at random). The excluded patients did not differ significantly from the included participants with respect to important aspects, including age, gender and severity of AS ( $\mathrm{P}$ value all $>0.2$ ).

\section{Biochemical analysis}

Plasma (EDTA) suPAR was analysed using a commercial CE/IVD (Conformité Européene /In Vitro Diagnostics) approved ELISA assay (suPARnostic, ViroGates, Copenhagen, Denmark). The assay was validated to measure suPAR levels between 0.6 and $22 \mathrm{ng} / \mathrm{mL}$. Samples were analysed at the same time point and in the same batch, with an interassay coefficient of variation of $9.9 \%$. SuPAR has been shown to be without substantial circadian variation, and the biomarker is stable in frozen samples. ${ }^{16}$

\section{Statistical analysis}

Data were analysed using IBM SPSS software V.24 and RStudio software V.0.98.1102 (RStudio). Continuous variables are presented as means \pm SD deviation for normally distributed values, median and IQR for skewed variables and numbers and percentages for categorical variables. Baseline suPAR was not associated with treatment allocation. Hence, the placebo and treatment groups were combined in a single cohort for analysis.

Non-normally distributed variables (including suPAR, EuroSCORE II and the STS risk score) were log2-transformed before analysis.

We evaluated the outcomes of AVR postoperative (all-cause) mortality and postoperative complications (defined as all-cause mortality, congestive heart failure, non-haemorrhagic or haemorrhagic stroke and renal impairment), occurring within the 30-day postoperative period. Cox analysis was performed for suPAR evaluated alongside the preoperative EuroSCORE II and the STS score. No further adjustment was made for confounders as both the EuroSCORE II and STS score are validated systems, which were deemed to have included relevant key variables. A further sensitivity analysis was performed incorporating the most recent preoperative suPAR values, which did not substantially alter the results.

The proportional hazards assumption for Cox's models was robust, as verified with plots of Schoenfeld residuals. The ability of the Cox models to predict adverse events was evaluated using standard metrics: C-statistic, continuous net reclassification index (NRI) and integrated discrimination index (IDI).

\section{Calculation of EuroSCORE II and STS risk score}

The calculation of EuroSCORE II and STS risk score used variables and definitions previously reported (online STS risk score calculator, V.2.81; http:/ / riskcalc. sts.org). ${ }^{9-11}$ The preoperative scores were calculated as part of a post hoc analysis using the most recent data available for each patient and included the following assumptions: New York Heart Association (NYHA) classification of heart failure was defined using established criteria, and though NYHA class was not explicitly recorded for each patient, it was inferred from recorded data (heart failure in conjunction with severity of dyspnoea). ${ }^{17}$ The insulin-dependent diabetes risk factor was assumed to be zero, given that pre-existing diabetes was an exclusion criterion in the SEAS study (four patients developed new-onset type 2 diabetes before AVR surgery and were assumed to be non-insulin dependent). The 'poor mobility' variable was assumed to be zero, as it was expected that the number of patients with poor mobility would be low in a study population free from major comorbidities at the onset of the study. Critical preoperative state was estimated based on documented arrhythmia (ventricular tachycardia or ventricular fibrillation), need for inotropes (one patient experienced a 'severe' third degree atrioventricular block, presumed to require inotropes) or acute renal failure (documented severe oliguria) occurring within a 3-day preoperative period (data was missing on preoperative aborted sudden death, cardiac massage, assisted ventilation, intra-aortic balloon or ventricular assist devices). Data on left main disease and immunosuppression treatment was unavailable for the STS score. All procedures were assumed to be elective. All patients gave written informed consent.

\section{RESULTS}

Baseline characteristics of the total study population (and subdivided by postoperative complications) are shown in table 1. During the 4.3-year follow-up period, for the 411 AVR procedures included in this study, 32 AVR postoperative complication events were recorded (including 14 
Table 1 Baseline characteristics of the total study population and subdivided by postoperative complications

\begin{tabular}{|c|c|c|c|}
\hline Variable & Total $(n=411)$ & $\begin{array}{l}\text { With postoperative } \\
\text { complications }(n=32)\end{array}$ & $\begin{array}{l}\text { Without postoperative } \\
\text { complications }(n=379)\end{array}$ \\
\hline \multicolumn{4}{|l|}{ Demographics } \\
\hline Age (years) & $67 \pm 9$ & $69 \pm 10$ & $67 \pm 9$ \\
\hline Female sex (\%) & 38 & 22 & 39 \\
\hline \multicolumn{4}{|l|}{ Haemodynamic parameters } \\
\hline Systolic BP (mm Hg) & $143 \pm 21$ & $137 \pm 21$ & $144 \pm 21$ \\
\hline Diastolic BP (mm Hg) & $82 \pm 11$ & $81 \pm 12$ & $82 \pm 11$ \\
\hline $\operatorname{LVEF}(\%)$ & $65 \pm 9$ & $63 \pm 9$ & $65 \pm 9$ \\
\hline \multicolumn{4}{|l|}{ Cardiovascular risk factors } \\
\hline Hypertension (\%) & 100 & 100 & 100 \\
\hline Current smoker (\%) & 21 & 28 & 20 \\
\hline BMI $\left(\mathrm{kg} / \mathrm{m}^{2}\right)$ & $27 \pm 4$ & $28 \pm 5$ & $27 \pm 4$ \\
\hline \multicolumn{4}{|l|}{ Laboratory values } \\
\hline SuPAR (ng/mL) & $2.6(2.1,3.3)$ & $3.0(2.5,3.9)$ & $2.6(2.1,3.2)$ \\
\hline Glucose (mmol/L) & $5.2(4.9,5.6)$ & $5.3(4.9,6.0)$ & $5.2(4.8,5.6)$ \\
\hline $\mathrm{CRP}(\mathrm{mg} / \mathrm{L})$ & $2.4(1.0,4.9)$ & $1.9(1.0,5.5)$ & $2.5(1.0,4.9)$ \\
\hline WBC $\left(10^{9} / L\right)$ & $6.0 \pm 1.6$ & $5.7 \pm 1.4$ & $6.1 \pm 1.6$ \\
\hline Total cholesterol (mmol/L) & $5.7 \pm 1.0$ & $5.3 \pm 0.8$ & $5.8 \pm 1.0$ \\
\hline LDL (mmol/L) & $3.5 \pm 0.9$ & $3.2 \pm 0.8$ & $3.6 \pm 0.9$ \\
\hline $\mathrm{HDL}(\mathrm{mmol} / \mathrm{L})$ & $1.6 \pm 0.4$ & $1.5 \pm 0.4$ & $1.6 \pm 0.4$ \\
\hline Triglycerides (mmol/L) & $1.2(0.9,1.7)$ & $1.2(0.9,1.4)$ & $1.2(0.9,1.7)$ \\
\hline Creatinine $(\mu \mathrm{mol} / \mathrm{L})$ & $94 \pm 16$ & $98 \pm 14$ & $93 \pm 16$ \\
\hline
\end{tabular}

BMI, body mass index; BP, blood pressure; CRP, C-reactive protein; HDL, high-density lipoprotein; LDL, low-density lipoprotein; LVEF, left ventricular ejection fraction; suPAR, soluble urokinase plasminogen activator receptor; WBC, white blood cells.

all-cause mortalities, eight strokes, eight incidences of renal failure and four incidences of congestive heart failure).

The ability of suPAR to predict postoperative mortality and complications, in relation to the EuroSCORE II and STS risk score models, is shown in table 2.
In univariate analysis, suPAR and STS score were both significantly associated with postoperative mortality and postoperative complications, while EuroSCORE II was only weakly associated.

\begin{tabular}{|c|c|c|c|c|}
\hline Model & $\begin{array}{l}\text { Postoperative mortality, HR } \\
(95 \% \mathrm{CI})\end{array}$ & $P$ value & $\begin{array}{l}\text { CEP of postoperative } \\
\text { complications, HR }(95 \% \mathrm{CI})\end{array}$ & P value \\
\hline \multicolumn{5}{|l|}{ Univariate } \\
\hline Log2-suPAR (ng/mL) & 3.5 (1.4 to 9.0$)$ & 0.008 & 2.7 (1.5 to 5.1 ) & 0.002 \\
\hline Log2-EuroSCORE II & $1.8(0.9$ to 3.7$)$ & 0.113 & $1.6(0.9$ to 2.6$)$ & 0.085 \\
\hline Log2-STS score & $2.4(1.2$ to 4.8$)$ & 0.013 & $1.7(1.0$ to 2.7$)$ & 0.032 \\
\hline \multicolumn{5}{|l|}{ suPAR/EurOSCORE II } \\
\hline Log2-suPAR (ng/mL) & 3.2 (1.2 to 8.6$)$ & 0.025 & 2.5 (1.3 to 5.0$)$ & 0.007 \\
\hline Log2-EuroSCORE II & $1.3(0.6$ to 2.8$)$ & 0.492 & $1.2(0.7$ to 2.0$)$ & 0.552 \\
\hline \multicolumn{5}{|l|}{ suPAR/STS score } \\
\hline Log2-suPAR (ng/mL) & 2.7 (1.0 to 7.8$)$ & 0.061 & 2.4 (1.2 to 4.8$)$ & 0.011 \\
\hline Log2-STS score & 1.9 (0.9 to 4.0$)$ & 0.104 & 1.3 (0.8 to 2.2$)$ & 0.263 \\
\hline
\end{tabular}

EuroSCORE II, European System for Cardiac Operative Risk Evaluation II; CEP, composite endpoint of postoperative complications (all-cause mortality, congestive heart failure, non-haemorrhagic or haemorrhagic stroke and renal impairment) occurring within the 30-day postoperative period; STS, Society of Thoracic Surgeons; suPAR, soluble urokinase plasminogen activator receptor. 
Table 3 Model performance (baseline univariate) and including suPAR for postoperative mortality and postoperative complications

\begin{tabular}{|c|c|c|c|c|c|c|}
\hline Model & C-statistic & $\begin{array}{l}\text { P value for } \\
\text { difference }\end{array}$ & NRI & $P$ value & IDI & $P$ value \\
\hline \multicolumn{7}{|l|}{ Postoperative mortality } \\
\hline Log2-EuroSCORE II & $0.55(0.35-0.74)$ & & & & & \\
\hline Log2-EuroSCORE II* & $0.69(0.50-0.89)$ & 0.103 & $38 \%$ & 0.048 & 0.018 & 0.034 \\
\hline Log2-STS score & $0.66(0.43-0.89)$ & & & & & \\
\hline Log2-STS score* & $0.71(0.51-0.91)$ & 0.056 & $29 \%$ & 0.088 & 0.019 & 0.032 \\
\hline \multicolumn{7}{|c|}{ Postoperative complications } \\
\hline Log2-EuroSCORE II & $0.58(0.44-0.72)$ & & & & & \\
\hline Log2-EuroSCORE II* & $0.68(0.56-0.81)$ & 0.085 & $30 \%$ & 0.024 & 0.022 & 0.012 \\
\hline Log2-STS score & $0.61(0.48-0.73)$ & & & & & \\
\hline Log2-STS score* & $0.67(0.56-0.79)$ & 0.058 & $36 \%$ & 0.008 & 0.021 & 0.012 \\
\hline
\end{tabular}

${ }^{*}$ Baseline model including suPAR.

EuroSCORE II, European System for Cardiac Operative Risk Evaluation II; IDI, integrated discrimination index; NRI, net reclassification index; STS, Society of Thoracic Surgeons; suPAR, soluble urokinase plasminogen activator receptor.

After mutually adjusting for suPAR and EuroSCORE II or STS score, suPAR remained associated with postoperative mortality and postoperative complications, although for the model containing suPAR and the STS score, the association of suPAR with postoperative mortality was of borderline significance.

\section{Discrimination tests}

The baseline models (using EuroSCORE II/STSscore alone) showed poor discrimination in predicting postoperative mortality and postoperative complications; however, inclusion of suPAR resulted in marginal (but non-significant) improvements in discrimination (as measured by the C-statistic) over the standard EuroSCORE II and STS score models, which translated into an improvement in the NRI and IDI indices (see table 3 ).

\section{DISCUSSION}

In initially asymptomatic patients with mild-moderate AS, we found that early detection of higher suPAR levels was of prognostic value in predicting 30-day postoperative mortality and postoperative complications after AVR, performed during a 4.3-year follow-up, and after adjusting for the EuroSCORE II or STS score. Inclusion of suPAR in the prediction models for postoperative complications or mortality, yielded significant improvements in the NRI, compared with the standard EuroSCORE II or STS score models. However, the discriminative power of the models was only modest.

Despite technological advances, standard AVR surgery remains a major procedure with the potential for several early complications, including valve related, cardiac non-valve related and non-cardiac events. The ability to risk-stratify individuals undergoing surgery and to recognise and treat potential complications is of clinical value, and efforts to incorporate various biomarkers in risk-stratification systems has met with some success. ${ }^{14}$
SuPAR has potential value as a biomarker due to its high stability in serum samples and limited circadian variation in plasma concentrations. ${ }^{16}$ At present, it remains unclear whether suPAR is merely a passive biomarker or a pathogenic agent. It has been suggested that suPAR is a causative factor in the development of chronic kidney disease and focal segmental glomerular sclerosis, although there is ongoing debate in the literature. ${ }^{2} 1819$ Higher plasma suPAR levels were demonstrated to be an early independent predictor of progressive renal dysfunction in patients with cardiovascular disease and normal kidney function, suggesting that suPAR may be a more sensitive marker of chronic kidney disease than traditional measures such as decline in estimated glomerular filtration rate or proteinuria. ${ }^{3}$ This is supported by a recent study, where suPAR predicted acute kidney injury in patients undergoing cardiac surgery, even in patients without pre-existing chronic kidney disease. ${ }^{6}$ Patients with chronic kidney disease are at increased risk of cardiovascular disease and death and are more vulnerable to acute kidney injury in the perioperative period..$^{20}$ Hence, we can speculate that early detection of elevated suPAR may identify patients with subclinical renal disease who presumably could benefit from closer monitoring to reduce the risk of complications. However, far from being limited to renal disease, suPAR has been associated with adverse outcomes in many studies and appears to reflect immune activation and inflammation in several disease states, including patients with cancer, critically ill patients and cardiovascular and liver disease. ${ }^{1-4}$ SuPAR has been implicated in many of the pathways involved in atherosclerosis, including the plasminogen activating pathway, inflammation, modulation of cell adhesion, migration and proliferation. ${ }^{22}$ Research suggests that suPAR represents a chronic atherosclerotic disease burden and subclinical organ damage, and suPAR has 
been demonstrated to be an independent predictor of incident cardiovascular disease and mortality in both 'healthy' individuals and in patients with established or suspected coronary artery disease. ${ }^{2324}$ Our results support the hypothesis that early detection of elevated suPAR levels may help identify a vulnerable patient, who is more prone to complications in the postoperative period. However, a precise cut-off for suPAR has yet to be established; hence, further research is required before suPAR can be adopted into current practice. Several limitations should be noted when interpreting these results. It is important to note that postoperative mortality and complications depend on both patient risk factors and care delivery factors, and technical problems during surgery as well as human and institutional factors in postoperative care, which have not been considered in our analysis. ${ }^{25}$ The SEAS study excluded patients with significant comorbidities (some of which are risk factors for AS progression); hence, it does not adequately represent the general population of patients with AS. A key limitation in this study is that preoperative suPAR levels were not explicitly recorded in the original study and brain natriuretic peptide measurements were not available. Preoperative EuroSCORE II and STS scores were estimated based on the most recent biochemistry data and involved several assumptions on the classification of NYHA class, insulin-dependent diabetes, critical perioperative state, pulmonary hypertension, poor mobility and urgency of the operation (see the Methods section). Missing data is likely to result in an underestimation of the true EuroSCORE II and STS scores for some patients. This study was a post hoc study, non-prespecified with the obvious limitations this carries.

\section{CONCLUSIONS}

SuPAR measured in patients with mild-moderate AS predicts postoperative mortality and complications in relation to later AVR surgery and may provide useful additive prognostic information to enable risk stratification of these patients. However, further prospective studies in other subsets of asymptomatic patients with AS are warranted before suPAR can be recommended for inclusion in risk prediction models for these patients.

\footnotetext{
Author affiliations

${ }^{1}$ Department of Medicine, Amager Hvidovre Hospital Glostrup, University of Copenhagen, Copenhagen, Denmark

${ }^{2}$ Department of Cardiology, Zealand University Hospital, Roskilde, Denmark ${ }^{3}$ Clinical Research Centre, Copenhagen University Hospital Hvidovre, Hvidovre, Denmark

${ }^{4}$ Department of Internal Medicine, Holbaek Hospital, Holbæk, Denmark ${ }^{5}$ Centre for Individualized Medicine in Arterial Diseases, Odense University Hospital, University of Southern Denmark, Odense, Denmark

${ }^{6}$ Research Unit, Skellefteå, Department of Public Health and Clinical Medicine, Umeå University, Umeå, Sweden

${ }^{7}$ Department of Cardiology, University Hospitals of South Manchester, Manchester Academic Health Sciences Centre, Manchester, UK
}

${ }^{8}$ Research Institute of Internal Medicine, Medical Research Center, Oulu University Hospital and University of Oulu, Oulu, Finland

${ }^{9}$ Department of Cardiology, Oslo University Hospital, Oslo, Norway

Acknowledgements The authors would like to thank the Novo Nordisk Foundation, whose research grant facilitated the writing of this article.

Contributors All authors made substantial contributions to the conception, design and analysis of this work and were involved in the revision and final approval of the version to be published. All authors have agreed to be accountable for all aspects of the work in ensuring that questions related to the accuracy or integrity of any part of the work are appropriately investigated and resolved.

Funding This study was partly funded by the Novo Nordisk Foundation.

Competing interests JEO is a co-founder and shareholder of ViroGates AS, Denmark, the company that produces the suPARnostic assay. He is a co-inventor on patents on suPAR and risk. Copenhagen University Hospital Hvidovre, Denmark, owns the patents, which are licensed to ViroGates AS. KB, SR, AYK and KW served on the SEAS Steering Committee were investigators and members of the steering committees of the SEAS study and have received honoraria from Merck \& $\mathrm{Co}$, the sponsor of the SEAS study. KB, SR and KW have received grant support from Merck $\&$ Co, the sponsor of the SEAS study. AYK reported Speaker's fee from Abbott, MSD and Novo Nordisk, Consult for MSD, research funding from MSD and ownership of Orion Pharma stock.

Ethics approval The ethical committees of all participating countries (Norway, Sweden, Denmark, Finland, Germany, the United Kingdom, and Ireland) approved the study (the SEAS study is registered at http://ClinicalTrials.gov, identifier NCT00092677).

Provenance and peer review Not commissioned; externally peer reviewed.

Data sharing statement № additional data are available.

Open Access This is an Open Access article distributed in accordance with the Creative Commons Attribution Non Commercial (CC BY-NC 4.0) license, which permits others to distribute, remix, adapt, build upon this work non-commercially, and license their derivative works on different terms, provided the original work is properly cited and the use is non-commercial. See: http://creativecommons.org/ licenses/by-nc/4.0/

(c) Article author(s) (or their employer(s) unless otherwise stated in the text of the article) 2018. All rights reserved. No commercial use is permitted unless otherwise expressly granted.

\section{REFERENCES}

1. Hodges GW, Bang CN, Wachtell K, et al. suPAR: a new biomarker for cardiovascular disease? Can J Cardiol 2015;31:1293-302.

2. Hayek SS, Koh KH, Grams ME, et al. A tripartite complex of suPAR, APOL1 risk variants and $\alpha v \beta 3$ integrin on podocytes mediates chronic kidney disease. Nat Med 2017;23:945-53.

3. Hayek SS, Sever S, Ko YA, et al. Soluble urokinase receptor and chronic kidney disease. N Engl J Med 2015;373:1916-25.

4. Zimmermann HW, Koch A, Seidler S, et al. Circulating soluble urokinase plasminogen activator is elevated in patients with chronic liver disease, discriminates stage and aetiology of cirrhosis and predicts prognosis. Liver Int 2012;32:500-9.

5. Hodges GW, Bang CN, Eugen-Olsen J, et al. SuPAR predicts cardiovascular events and mortality in patients with asymptomatic aortic stenosis. Can J Cardiol 2016;32:1462-9.

6. Mossanen JC, Pracht J, Jansen TU, et al. Elevated soluble urokinase plasminogen activator receptor and proenkephalin serum levels predict the development of acute kidney injury after cardiac surgery. Int $J$ Mol Sci 2017;18:1662.

7. Brown ML, Pellikka PA, Schaff HV, et al. The benefits of early valve replacement in asymptomatic patients with severe aortic stenosis. $J$ Thorac Cardiovasc Surg 2008;135:308-15.

8. Rosenhek R, lung B, Tornos P, et al. ESC working group on valvular heart disease position paper: assessing the risk of interventions in patients with valvular heart disease. Eur Heart J 2012;33:822-8.

9. Nashef SA, Roques F, Sharples LD, et al. EuroSCORE II. Eur J Cardiothorac Surg 2012;41:734-45.

10. O'Brien SM, Shahian DM, Filardo G, et al. The society of thoracic surgeons 2008 cardiac surgery risk models: part 2 - isolated valve surgery. Ann Thorac Surg 2009;88:S23-S42.

11. Shahian DM, O'Brien SM, Filardo G, et al. The society of thoracic surgeons 2008 cardiac surgery risk models: part 3 - valve 
plus coronary artery bypass grafting surgery. Ann Thorac Surg 2009;88:S43-S62.

12. Monin JL, Lancellotti $\mathrm{P}$, Monchi $\mathrm{M}$, et al. Risk score for predicting outcome in patients with asymptomatic aortic stenosis. Circulation 2009;120:e75-75.

13. Holme I, Pedersen TR, Boman $\mathrm{K}$, et al. A risk score for predicting mortality in patients with asymptomatic mild to moderate aortic stenosis. Heart 2012;98:377-83.

14. Lindman BR, Breyley JG, Schilling JD, et al. Prognostic utility of novel biomarkers of cardiovascular stress in patients with aortic stenosis undergoing valve replacement. Heart 2015;101:1382-8.

15. Rossebø AB, Pedersen TR, Allen C, et al. Design and baseline characteristics of the simvastatin and ezetimibe in aortic stenosis (SEAS) study. Am J Cardiol 2007;99:970-3.

16. Andersen O, Eugen-Olsen J, Kofoed K, et al. Soluble urokinase plasminogen activator receptor is a marker of dysmetabolism in HIVinfected patients receiving highly active antiretroviral therapy. J Med Virol 2008;80:209-16.

17. Criteria Committee of the New York Heart Association. Nomenclature and criteria for diagnosis of diseases of the heart and great vessels. 9th edn. Boston, Massachusetts: Little, Brown \& Co, 1994:253-6.

18. Peev V, Hahm E, Reiser J. Unwinding focal segmental glomerulosclerosis. F1000Res 2017;6:466.
19. Gallon L, Quaggin SE, SuPAR QSE. SuPAR and FSGS: is the jury still out? Nat Rev Nephrol 2017;13:593.

20. Gansevoort RT, Correa-Rotter R, Hemmelgarn BR, et al. Chronic kidney disease and cardiovascular risk: epidemiology, mechanisms, and prevention. Lancet 2013;382:339-52.

21. Borthwick E, Ferguson A. Perioperative acute kidney injury: risk factors, recognition, management, and outcomes. BMJ 2010;341:c3365.

22. Thunø M, Macho B, Eugen-Olsen J. suPAR: the molecular crystal ball. Dis Markers 2009;27:157-72.

23. Eugen-Olsen J, Andersen O, Linneberg A, et al. Circulating soluble urokinase plasminogen activator receptor predicts cancer, cardiovascular disease, diabetes and mortality in the general population. J Intern Med 2010;268:296-308.

24. Eapen DJ, Manocha P, Ghasemzadeh N, et al. Soluble urokinase plasminogen activator receptor level is an independent predictor of the presence and severity of coronary artery disease and of future adverse events. J Am Heart Assoc 2014;3:e001118.

25. Westaby S, De Silva R, Petrou M, et al. Surgeon-specific mortality data disguise wider failings in delivery of safe surgical services. Eur $J$ Cardiothorac Surg 2015;47:341-5. 